\title{
Dynamique spatio-temporelle de la végétation et des simulies vecteurs de l'onchocercose cécitante au Bénin
}

\author{
M.C. HOUEVOGANWA ${ }^{1}$, C. OUINSAVI ${ }^{2 *}$, E. GOUDEGNON ${ }^{3}$, C. GBEMAVO ${ }^{2}$, \\ N. SOKPON ${ }^{2}$ et S. AKPONA ${ }^{4}$ \\ ${ }^{1}$ Laboratoire d'Analyses Biomédicales, CHD/Borgou, BP : 02 Parakou, Bénin. \\ ${ }^{2}$ Laboratoire d'Etudes et de Recherches Forestières, Université de Parakou, \\ Faculté d'Agronomie, BP : 123. Parakou, Bénin. \\ ${ }^{3}$ Ecole Normale Supérieure de Natitingou, Université de Parakou, BP : 72. Natitingou, Bénin. \\ ${ }^{4}$ UERS de Biochimie et de Biologie Moléculaire, Faculté de Médecine, \\ Université de Parakou. BP : 123. Parakou, Bénin. \\ *Auteur correspondant, E-mail : ouinsch@yahoo.fr
}

\section{RESUME}

La dynamique spatio-temporelle des simulies, vecteurs de l'onchocercose cécitante en fonction de la dynamique de la végétation a été étudiée dans quatre localités bioécologiques de son aire de répartition au Bénin entre les années 1990 et 2010. Il ressort de ces travaux que l'effectif des simulies (Simulium damnosum) a augmenté par région dans le temps consécutif au recul temporel du couvert forestier. Les simulies savanicoles porteuses des larves cécitantes ont accru ces dernières années dans chacune des régions contrairement aux simulies forestières avec un accent particulier dans la zone Soudano-guinéenne. La nuisance simulidienne et la transmission de l'onchocercose cécitante varient d'une région à une autre $(\mathrm{P}<0,001)$. Les régions de la zone Soudano-guinéenne sont beaucoup plus exposées à la résurgence de l'onchocercose cécitante (PMT moyen élevée) par rapport à celle de la zone Guinéenne.

(C) 2014 International Formulae Group. All rights reserved.

Mots clés : Forêts, savane, simulies, environnement, santé, Bénin.

\section{INTRODUCTION}

Les maladies à transmission vectorielle représentent $17 \%$ des maladies infectieuses et figurent parmi les plus importantes pathologies en santé humaine, tant par la morbidité que par la mortalité qu'elles entraînent (Poinsignon, 2008). Ces maladies sont principalement répandues dans les régions tropicales où elles sont à la fois les causes et les conséquences de sous- développement. Leur impact en santé publique constitue un frein à l'éducation des enfants et au développement économique des pays. Ces maladies constituent pour les hommes des menaces constantes de santé publique et souvent multiples dans de vastes zones géographiques. Le climat tropical de l'Afrique et particulièrement celui de l'Afrique de l'Ouest est favorable à la plupart des grandes maladies à transmission 
vectorielle comme le paludisme, la schistosomiase, l'onchocercose, la trypanosomiase, la filariose, la leishmaniose, la peste, la fièvre de la Vallée du Rift, la fièvre jaune et les fièvres hémorragiques transmises par les tiques. Des espèces vectrices d'Afrique se sont adaptées à des écosystèmes allant de la forêt ombrophile à la savane sèche et toute modification de ces écosystèmes entraînera une modification de la distribution des espèces vectrices (Andrew et al., 2001; Mouchet et Carnevale, 1997). L'Afrique présente donc une grande diversité de complexes d'espèces vectrices qui ont la possibilité de se redistribuer dans de nouveaux habitats en fonction de leurs conditions bioécologiques, ce qui peut entraîner une nouvelle répartition des maladies.

Dans son rapport portant sur l'évaluation des ressources forestières mondiales, l'Organisation des Nations Unies pour l'Alimentation et l'Agriculture (FAO), note que, bien qu'il y ait un recul de la déforestation durant ces dix dernières années grace aux programmes nationaux de reboisement et de la législation sur les forêts, la déforestation se poursuit à un rythme alarmant dans certaines régions du monde (Amérique du Sud, Afrique et Océanie) (FAO, 2010). S'agissant de l'Afrique, le continent aurait perdu environ 4 millions d'hectares de forêt par an de 2000 à 2005, soit près d'un tiers de la superficie déboisée dans le monde. Entre 2000 et 2010, l'Afrique aurait perdu 3,4 millions d'hectares de forêts (FAO, 2009). En Afrique de l'Ouest et particulièrement au Bénin, les maigres ressources forestières disponibles ces dernières années sont le résultat d'une dégradation des forêts par l'homme depuis plusieurs décennies et que de nos jours, les pressions humaines sur les forêts sont encore très fortes. Cette dynamique spatio-temporelle de la végétation due surtout aux activités anthropiques (abattage de la forêt pour des fins d'agricultures, l'exploitation forestier et les feux de végétation) affectera donc la biologie et l'écologie des vecteurs et des hôtes intermédiaires. Ce qui a pour corollaire une augmentation du risque d'extension de nombreuses maladies à transmission vectorielle. Or concernant l'onchocercose humaine, des études comparatives en Afrique de l'Ouest, conduites par différents auteurs indiquent que les manifestations cliniques de la maladie sont beaucoup plus sévères en savane qu'en forêt (Philippon, 1977; Quillévéré, 1979 ; Monjusiau et al., 1965; Budden, 1963 ; Anderson et al., 1974). Cette différence clinique de la maladie est due au fait que les souches d'onchocerques de savane, transmises par des simulies vectrices savanicoles, sont plus pathogènes pour l'homme que les souches de forêt transmises par des simulies forestières. Ces vecteurs appartiennent au complexe Simulium damnosum au sein duquel les espèces sont bien différenciées du point de vue cytotaxonomique ainsi que par leur bioécologie et capacité vectrice (Doukouré et al., 1994). Il est donc nécessaire et indispensable d'évaluer les dangers de la dynamique spatiotemporelle de la forêt pour le comportement des espèces forestières et/ou savanicoles de simulies; des effets de la survie d'espèces forestières dans la savane et vice-versa et sur leur interaction.

Au Bénin, on ne dispose pas encore des données sur l'effet de la dynamique spatiotemporelle de la végétation sur la nuisance simulidienne et sur la transmission de la maladie. En Afrique, certains travaux ont été réalisés sur le sujet notamment ceux de Klein et Traoré au Cameroun sur la nuisance simulidienne dans la région de Makondo. L'objectif de ce travail est d'étudier la dynamique spatio-temporelle de la végétation et des simulies, vecteurs de l'onchocercose cécitante en zone Guinéenne et SoudanoGuinéenne de transition au Bénin afin de mettre en lumière d'éventuelles tendances futures de l'impact de ce phénomène alarmant sur la nuisance simulidienne et sur la transmission de l'onchocercose cécitante, en République du Bénin. 


\section{MATERIEL ET METHODES}

Milieu d'étude et choix des sites d'étude

Le Bénin est un petit pays de l'Afrique occidentale, couvrant une superficie de $112622 \mathrm{~km}^{2}$, s'étendant entre $1^{\circ}$ et $30^{\circ} 40^{\prime}$. Elle est limitée au Nord par la République du Niger, au Nord Ouest par le Burkina Faso, à l'Est par la République Fédérale du Nigéria, à l'Ouest par le Togo et au Sud par l'Océan Atlantique.

La présente étude s'est déroulée globalement au Centre du Bénin, c'est-à-dire entre les parallèles $6^{\circ} 55^{\prime}$ et $8^{\circ} 50^{\prime}$ de latitude Nord et les méridiens $1^{\circ} 58^{\prime}$ et $2^{\circ} 45^{\prime}$ de longitude Est (Figure 1) (Capo-chichi, 2006). Cette étendue correspond à une partie de l'aire de répartition des simulies, vecteurs de l'onchocercose au Bénin et conformément aux exigences bioécologiques de l'espèce. Quatre localités ont été choisies dans cette zone à savoir : Kaboua et M'bétékoukou dans la zone Soudano-Guinéenne; Agonlin-Pahou et Atchérigbé dans la zone Guinéenne (Figure 1). La zone Guinéenne ou Subéquatoriale est caractérisée par une pluviométrie variant de 900 à $1200 \mathrm{~mm}$ par an. On distingue quatre saisons dont deux humides. La température moyenne annuelle y est de $26{ }^{\circ} \mathrm{C}$ et l'humidité moyenne annuelle est de $75 \%$. La zone soudano-guinéenne est située entre $7^{\circ}$ et $10^{\circ}$ de latitude Nord avec des fluctuations de température très marquées et une pluviométrie annuelle de $1200 \mathrm{~mm}$. Elle est une zone de transition au sein de laquelle la seconde saison humide tend à disparaître. Ce qui aura pour conséquence à terme, le remplacement de cette zone soudano-guinéenne par une zone soudanienne avec cinq mois secs. La température moyenne y est de $27^{\circ}$ et l'humidité relative est annuellement voisine de $60 \%$. La végétation est caractérisée de façon générale par des vastes savanes boisées, arborées et arbustives au niveau desquelles serpentent quelques forêts galeries le long des cours d'eaux et quelques rares forêts denses. Les savanes boisées et savane arborée sont caractérisées par les essences végétales telles que : Vitellaria paradoxa, Parkia biglobosa, Combretum spp, Monotes kerstingii, Terminalia spp, Daniellia oliveri, Lophira lanceolata, Mitragyna inermis et de Acacia polyacantha, Isoberlinia doka et I. tomentosa tandis que les forêts denses et les galeries forestières sont caractérisées par les essences telles que Terminalia superba, Triplochiton scleroxylon, Milicia excelsa, Antiaris toxicaria, Nothospondias staudtii, Parinari spp., Pterocarpus santalinoides, Cola gigantea, Berlinia grandiflora (Agbahoungba et al., 2001). Les principales activités sont caractérisées par :

L'agriculture grâce surtout aux sols hydromorphes retrouvés dans les vallées et bas fonds.

L'élevage et des échanges commerciaux à cause de la proximité du Nigéria à la zone d'étude qui joue un rôle très important dans la distribution de la maladie.

\section{Espèce étudiée}

La simulie cécitante de l'onchocercose (Simulium damnosum) est l'espèce étudiée. Elle est du règne animal, de l'embranchement des arthropodes, de la classe des insectes, de l'ordre des diptères, de la famille des Simulidaes et du genre Simulium (Harcel, 2008). L'espèce retrouvée dans la zone d'étude au Bénin est Simulium damnosum s.s. dite de savane et les espèces du sous complexe S. Sanctipauli et du sous complexe $S$. Squamosum dites de forêt. Le complexe $S$. damnosum a des exigences particulières qui conditionnent l'établissement des gîtes préimaginaux. Certaines conditions sont indispensables et doivent être remplies simultanément pour que se forme et se maintienne un gîte larvaire de $S$. damnosum s.s. (Le Berre, 1966) : une eau douce courante d'une vitesse comprise entre 0.30 et $1.5 \mathrm{~m} / \mathrm{s}$., la présence de supports naturels ou artificiels, une eau très oxygénée, une eau riche en matières organiques en suspension, un $\mathrm{pH}$ tolérable $(\cong 7$ ). Les trois facteurs principaux qui rendent le mieux compte de la répartition des gîtes larvaires des différentes espèces du 
complexe $S$. damnosum sont: le $\mathrm{pH}$, la conductivité, et la température des eaux des gîtes larvaires (Quillévéré, 1979). En effet en saison sèche, les différentes espèces de simulies présentent des comportements différents face à ces trois facteurs. Au stade adulte, les simulies présentent des préférences d'ordre climatique. Un milieu favorable doit présenter une hygrométrie élevée et des zones d'ombre (Nuage ou couvert végétal). La température est également un facteur limitant. Les températures minimales et maximales tolérées dépendent de l'âge de la femelle (Léonard, 1996).

\section{Collecte des données sur la dynamique simulidienne}

Afin d'évaluer le niveau de la prolifération des simulies, la dynamique des simulies savanicoles et forestières et le risque de recrudescence de l'onchocercose; la capture, l'identification et la dissection des simulies ont été effectuées. La technique classique de capture de simulies sur appât humain a été utilisée au cours de notre étude. Les captureurs, jambes dénudées jusqu'aux genoux, récoltent dans des tubes à hémolyse classiques (65 $\mathrm{mm}$ de long) toutes les simulies. Les captures ont lieu de 7 à 18 heures et à raison de 3 fois par semaine pendant 3 mois (juillet, août et septembre), à l'abri du vent et du soleil. Une équipe composée de deux personnes assurait la capture des simulies, chacun travaillant à tour de rôle pendant une heure. Les différentes espèces du $S$. damnosum ont été identifiées à l'aide de caractères fondés sur l'observation de la teinte des touffes de soies situées à la base des ailes, de la teinte relative des antennes, de la procoxa, du prosternum, et du mesonotum (Soungalo et al., 2006). Cette identification des simulies permet premièrement de les classer dans le groupe de Simulium damnosum et deuxièmement dans le groupe des simulies forestières et savanicoles.

La dissection des simulies a permis premièrement de savoir si les simulies étaient jeunes et nées localement ou plus âgées. Cela est déterminé en comptant le nombre de mouches (simulies) qui avaient atteint le stade ovipare de leurs vies. Deuxièmement, l'évaluation de l'intensité de la transmission potentielle a été faite à partir de la dissection des simulies femelles adultes, du comptage du nombre de simulies infestantes et $\mathrm{du}$ nombre de larves infestieuses d'Onchocerca volvulus retrouvé dans la tête des simulies (simulies infestieuses).

\section{Collecte des données sur la dynamique d'occupation du sol}

La dynamique d'occupation des sols de la zone de l'étude a été étudiée sur les variations de deux images satellitaires Landsat des années 1990 et 2008 et du fond topographique de la zone obtenues auprès du Centre National de Télédétection et de surveillance du couvert forestier (CENATEL) et de l'Institut Géographique Nationale (IGN). Les images qualifiées de hautes (10$30 \mathrm{~m})$ résolutions spatiales ont été prétraitées en suivant les étapes et opérations ci - après:

o Le Géoréférencement qui est la détermination de l'ellipsoïde (datum) et du système de coordonnées cartésiennes. Il a été réalisé après scannage du fonds topographique ;

○ Le redressement géométrique qui est la correction géométrique de l'image obtenue après la première opération de digitalisation tout en tenant compte de la courbure du globe terrestre ;

- Le lissage qui consiste à débarrasser les images des incorrections causées par l'angle d'observation, les conditions d'éclaircissement et les effets atmosphériques ;

- La composition colorée qui consiste à classer automatiquement les valeurs radiométriques de chaque image en des classes d'unités identiques ;

○ Le contrôle de terrain a permis de vérifier les emplacements des unités géomorphologiques et biogéographiques sur la carte thématique à la réalité du terrain ; 
- La clarification de l'interprétation supervisée qui est faite de façon visuelle à l'ordinateur après échantillonnage, ce qui conduit à l'évaluation statistique et enfin la légende puis la mise en page.

La Figure 2 illustre le processus de traitement et d'interprétation d'images satellitaires.

Traitement statistique des données entomologiques et épidémiologiques

Les différents indices entomologiques et épidémiologiques à savoir: le Taux hebdomadaire de piqûre (THP), le Taux mensuel de piqûre $($ TMP $=$ MBR : Monthly Biting Rate), le Potentiel Hebdomadaire de Transmission (PHT) et le Potentiel Mensuel de Transmission (PMT), le Taux de Simulies Infectées (TSI) et les différents écosystèmes à savoir le nombre total de simulies savanicoles et le nombre total de simulies forestières sont soumis à une analyse de variance, et à une analyse en composante principale (ACP) afin d'analyser l'effet de chacun de ces facteurs et leurs interactions sur la transmission de l'onchocercose dans les différentes localités de la zone d'étude. Le THP et le TMP permet d'apprécier la densité des simulies présentes dans la zone d'étude tandis que le PHT et le PMT permet d'apprécier l'intensité de la transmission potentielle. Le logiciel statistique minitab 14 a été utilisé à cet effet. La période de l'étude a été subdivisée en trois temps : T1 $=1990$ à $1994 ; \mathrm{T} 2=1995$ à 1999 et T3 = 1999 à 2010. Les différents indices entomologiques et épidémiologiques ont été calculés à partir des formules ci-après :

$\mathrm{THP}=\frac{\mathrm{NSC} \times \mathrm{NJ} / \mathrm{S}}{1}$

Avec :

NSC $=$ Nombre de Simulies Capturées

$\mathrm{NJ} / \mathrm{S}=$ Nombre de Jours/Semaine

$$
\text { MBR }=\text { TMP }=\frac{\sum \text { THPX le NSM }}{\text { NsM }}
$$

Avec :

NSM= Nombre de Semaine dans le Mois

NsM= Nombre de sortie dans le Mois

PHT $=\frac{\text { NSC } \times 7 \times \text { NLT3 })}{1 \times \text { NJC } \times \text { NSD }}$

Avec :

NSC := Nombre de Simulies Capturées

NLT3 $=$ Nombre de Larves de stade 3 dans la

tête

NJC $=$ Nombre de Jours de Capture

NSD $=$ Nombre de Simulies Disséquées

$P M T=\frac{\sum \text { PHT X NSM }}{\text { NsM }}$

Avec :

$\mathrm{NSM}=$ Nombre de Semaines dans le Mois

$\mathrm{NsM}=$ Nombre de sorties dans le Mois.

Analyses statistiques de la dynamique spatiale d'occupation des terres

Dans le cadre de la présente étude, les superficies des différentes unités d'occupation ont été calculées à partir du croisement ou de l'intersection des cellules de l'occupation des terres à deux dates (1990 et 2008), à l'aide de l'algorithme Intersect polygons de l'extension Geoprocessing du logiciel Arcview 9.2; ce qui a permis une comparaison de ces différentes unités de ces années.

Soit $S_{1}$ et $S_{2}$ les surfaces occupées par une même identité (forêts denses par exemple) au cours des années 1 et 2 .

Si $S_{2}-S_{1}=$ nul, cela signifie une stabilité de la superficie des forêts denses de l'année 1à 2 ;

Si $S_{2}-S_{1}=$ négatif, cela signifie une régression de la superficie des forêts denses de l'année 1 à 2 ;

Si $S_{2}-S_{1}=$ positif, cela signifie une augmentation de la superficie des forêts denses de l'année 1 à 2. 


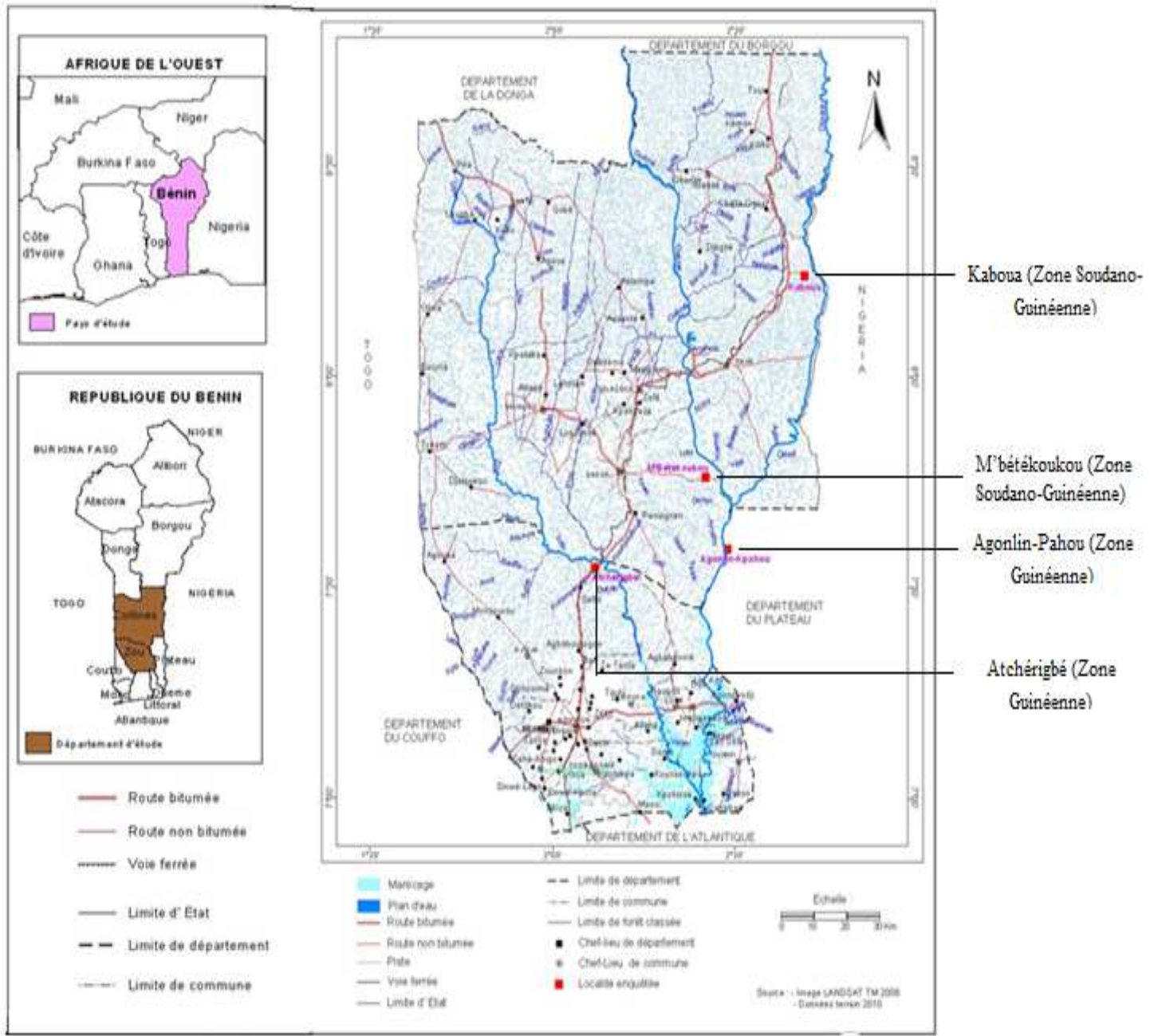

Figure 1 : Localisation des foyers d'étude dans les zones Guinéennes et Soudano-Guinéennes.

\section{RESULTATS}

Le Tableau 1 présente les formes d'occupation des terres dans la zone d'étude en 1990, 2008 et leurs variations en 18 ans. L'analyse du Tableau 1 révèle un recul significatif de : la galerie forestière, la forêt dense, la forêt claire et savane boisée, la savane arborée et arbustive et des cultures et jachères à palmier au profit des cultures et des jachères, des plantations et des agglomérations.

Cette dynamique spatio-temporelle de la végétation serait due aux activités anthropiques: abattage de la forêt pour des fins d'agriculture, l'exploitation forestier et les feux de végétation. Elle est responsable de l'invasion des zones par les simulies car facilite leur capacité de dispersion. En effet les simulies sont aidées par les mouvements des vents, en occurrence le Front Intertropical (FIT) qui ne rencontre plus assez de difficultés au cours de son déplacement avec les simulies à la recherche de repas sanguin (simulies femelles surtout). De plus, cette régression pourrait aussi avoir des effets sur les caractéristiques physico-chimiques des eaux des gîtes larvaires (facteurs limitant des larves préimaginaux des différentes espèces de Simulium damnosum) favorisant l'installation 
d'autres espèces de simulies autres que celles retrouvées habituellement.

\section{Evaluation spatio-temporelle de la prolifération des simulies et de la transmission de l'onchocercose}

Le Tableau 2 présente les variations dans le temps du nombre total moyen de simulies, des indices entomologiques et épidémiologiques par localité et par écosystème dans le temps. Par localité, elle est de 4,86 pour le nombre total moyen de simulies; 13,71 pour le taux mensuel de piqûre ; 8,09 pour le Potentiel Mensuel de Transmission. Par période, elle est de 31,74 pour le Taux Mensuel de Piqûre (TMP) et par interaction localité-période, elle est de 2,52. L'analyse de variance de l'évaluation spatiotemporelle de la prolifération des simulies et de la transmission de l'onchocercose révèle une différence hautement significative $(\mathrm{P} \leq 0,001)$ de l'évolution du taux mensuel de piqûre, du potentiel mensuel de transmission et du nombre total moyen de simulies en fonction de la région et de la période. Par ailleurs, seul l'effet de l'interaction de la région et de la période sur le Potentiel Mensuel de Transmission (PMT) est significatif (Tableau 2). Cela implique que l'influence du facteur localité sur le MTP dépend du facteur période et vice-versa. Toutes les autres interactions des facteurs sur la prolifération des simulies n'ont pas d'effet significatif.

La Figure 3 présente les modalités des facteurs période et localité sur le Taux Mensuel de Piqûre (MBR). Les résultats d'analyse de l'interaction entre les modalités des deux facteurs (Localité et Période) sur le MBR (Figure 3) révèlent qu'au cours des deux premières périodes et suivant les localités, la variation du MBR moyen reste très faible. Cet indice est plus élevé en moyenne dans la zone Soudano-guinéenne que dans la zone Guinéenne. Pendant la dernière période T2 à T3, le MBR moyen des quatre localités a augmenté de façon drastique dans l'ensemble des régions et a dépassé le seuil annuel à partir duquel la nuisance simulidienne devient intolérable dans une zone, donc un problème de Santé publique. La prolifération des simulies est donc beaucoup plus intense dans les localités du domaine Soudano-Guinéenne que celles du domaine Guinéen.

La Figure 4 présente les modalités des facteurs période, localité et écosystèmes constitués par le nombre total moyen de simulies forestières et celui de simulies savanicoles. L'interaction entre les modalités des trois facteurs (localités, périodes, écosystèmes) sur la prolifération des simulies révèle que dans le temps, la prolifération des simulies savanicoles et forestières a été plus intense dans la région soudano-guinéenne que celle de la région guinéenne. Les simulies forestières ont diminué dans le temps, tandis que les simulies savanicoles ont diminué entre les années 1990 à 1999 puis ont dangereusement augmenté de 1999 à 2010 (Figure 4).

Le Tableau 3 présente le nombre total moyen de simulies et les indices entomologiques et épidémiologiques par localité. Le nombre total moyen de simulies est respectivement de $360 ; 239 ; 1052$ et 891 à Agonlin-Pahou, Atchérigbé, Kaboua et M'bétékoukou. Leurs taux mensuels de piqûre sont respectivement de $3256 ; 2584 ; 9669$ et 8763 tandis que leurs Potentiels Mensuels de Transmissions sont respectivement de 146,$42 ; 47,92 ; 321,12 ; 426,14$. Le nombre de simulies forestières est en moyenne de 244 à Agonlin-Pahou, 64 à Atchérigbé, 413,63 Kaboua et 602 à M'bétékoukou alors que le nombre de simulies savanicole est en moyenne de $116 ; 175 ; 638$ et 289 respectivement dans ces différentes localités.

L'analyse de ces différents résultats révèle que la prolifération des simulies est plus intense dans le domaine SoudanoGuinéen de Transition que dans le domaine Guinéen; ce qui confirme la forte nuisance simulidienne subie surtout par les riverains de la localité du domaine Soudano-Guinéen contrairement à ceux de la localité du domaine Guinéen. De plus, le potentiel de transmission de la maladie est plus élevé dans le domaine 
Soudano-Guinéen de Transition avec une forte prolifération de simulies savanicoles.

Structuration des régions en fonction du nombre total moyen de simulies et des indices entomologiques et épidémiologiques

La Figure 5 présente la structuration des différentes localités enquêtées dans un système d'axes en fonction du nombre total moyen de simulies, du Taux Mensuel de Piqûre (MBR), de la pluviométrie, du taux de simulies infectées et du Potentiel Mensuel de Transmission PMT). Les résultats d'analyse de la structuration des localités en fonction de ces différents paramètres révèlent que $94,2 \%$ des informations contenues dans les variables sont contrôlées par les deux premiers axes. Les localités se trouvant du côté positif du premier axe sont caractérisées par une hauteur moyenne de pluie élevée, une densité de simulies présentes, et une intensité de transmission faible. Les populations de ces régions, surtout celles riveraines, subissent alors moins de nuisance simulidienne. Il s'agit des régions Agonlin-Pahou et Atchérigbé (Figure 5). Les localités de M'bétékoukou et Kaboua (Zone soudanoGuinéenne) s'opposent aux précédentes suivant ces caractéristiques et confirment donc les résultats précédents. L'axe 1peut être interprété comme symbolisant le gradient pluviométrique.

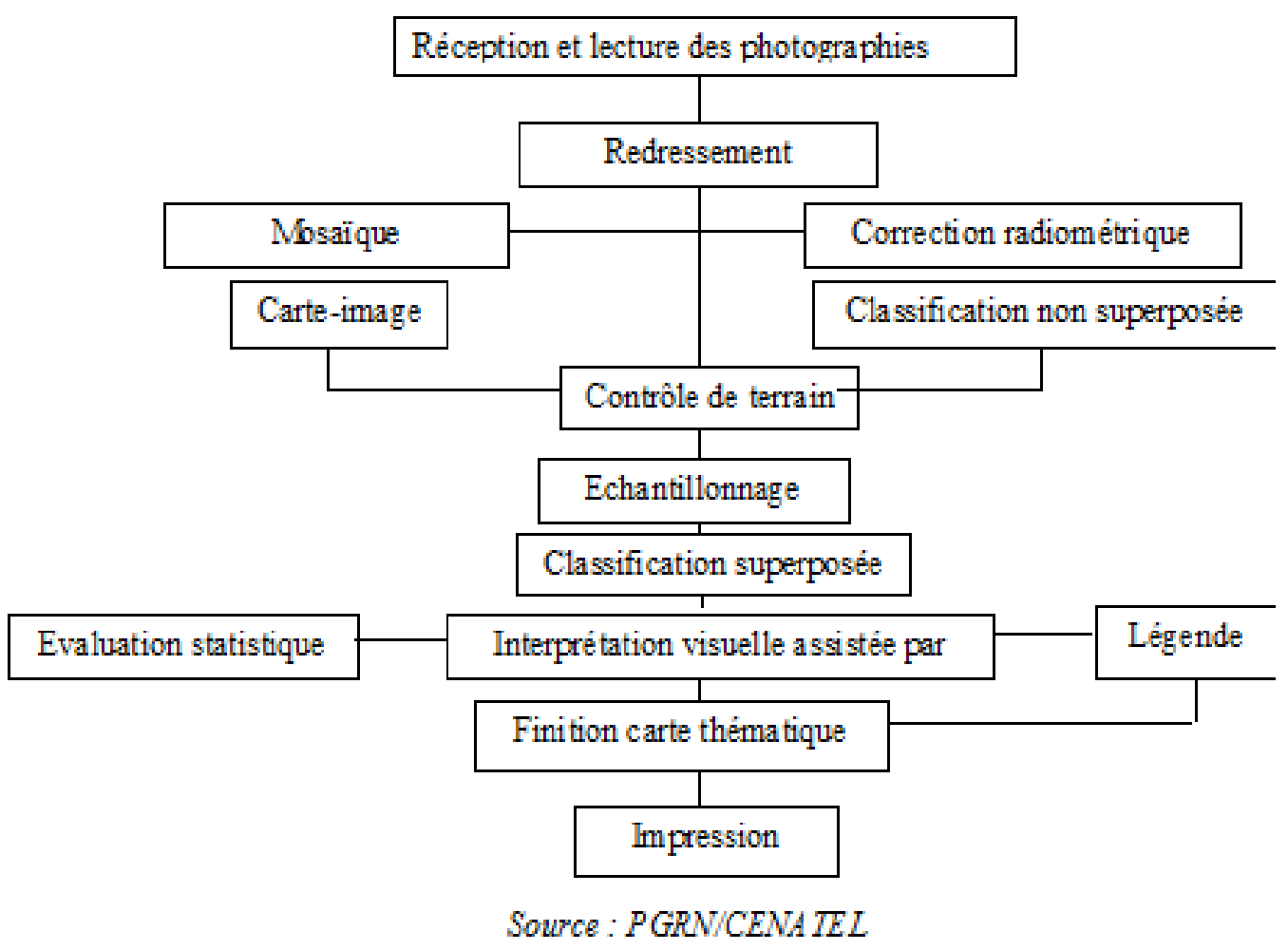

Figure 2 : Processus de traitement et d'interprétation d'image. 


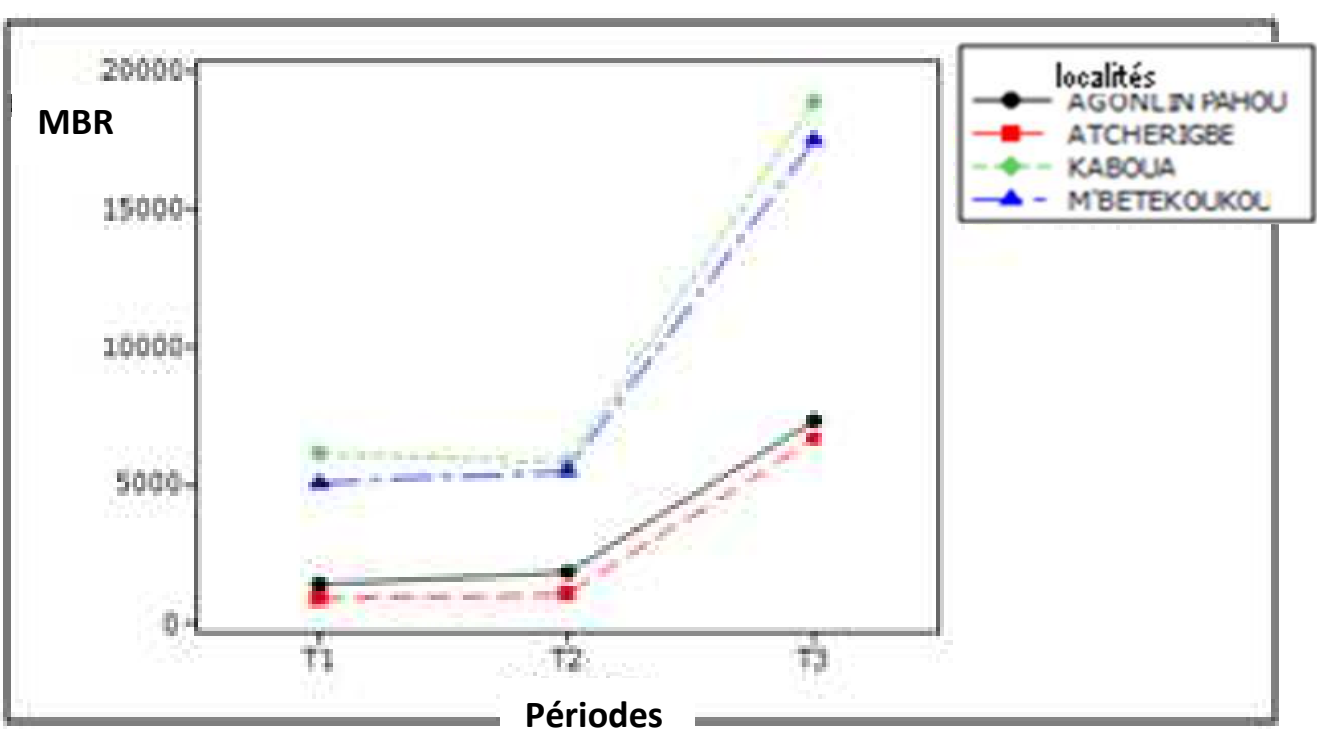

Figure 3 : Interaction entre les modalités des deux facteurs (Localité, ) sur le . T1 = 1990 à 1994 T2 = 1995 à 1999 T3 = 2007 à 2010 MBR = Monthly Biting Rate.

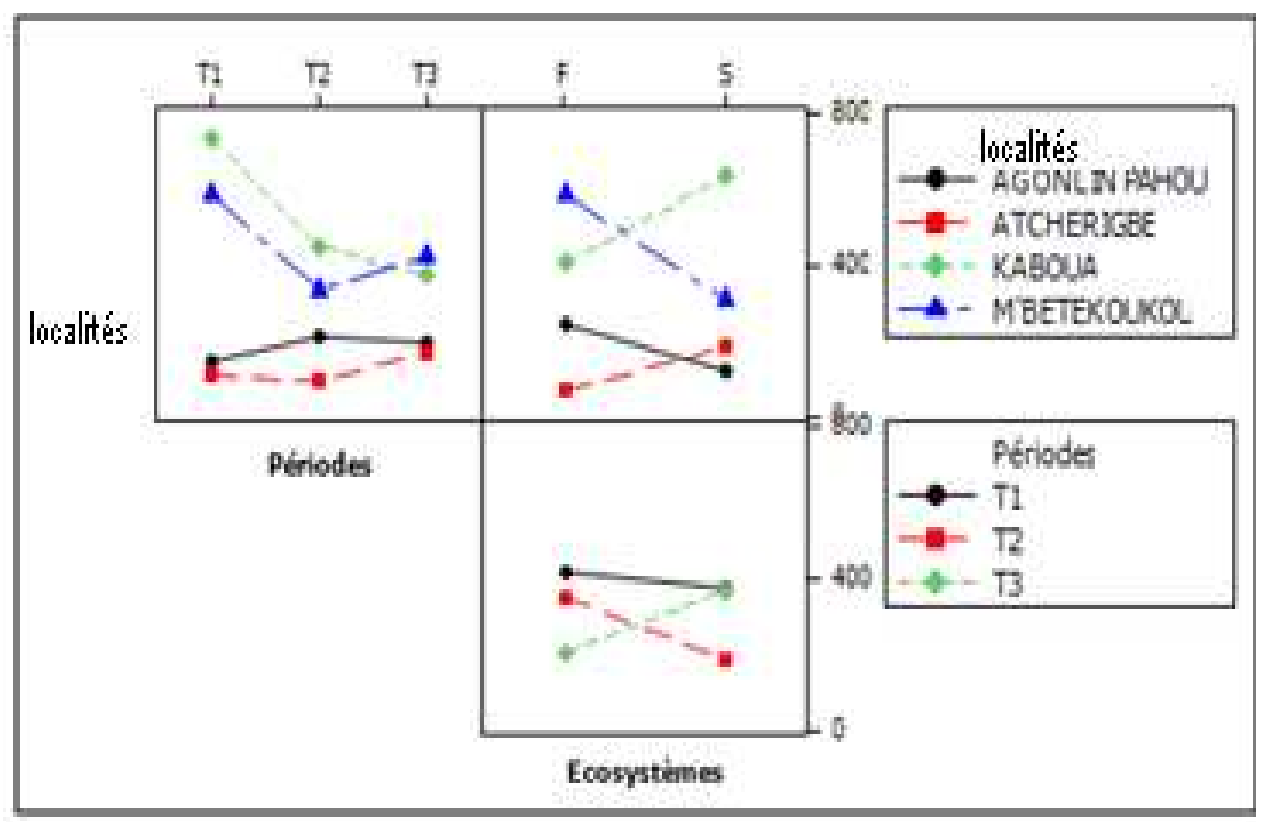

Figure 4: Interaction entre les modalités des trois facteurs (Régions, Période, Ecosystèmes) sur la prolifération des simulies. T1 = 1990 à $1994 \quad \mathrm{~T} 2=1995$ à $1999 \quad \mathrm{~T} 3=2007$ à $2010 \quad \mathrm{~F}=$ nombre de simulies forestière $\mathrm{S}=$ nombre de simulies savanicole 


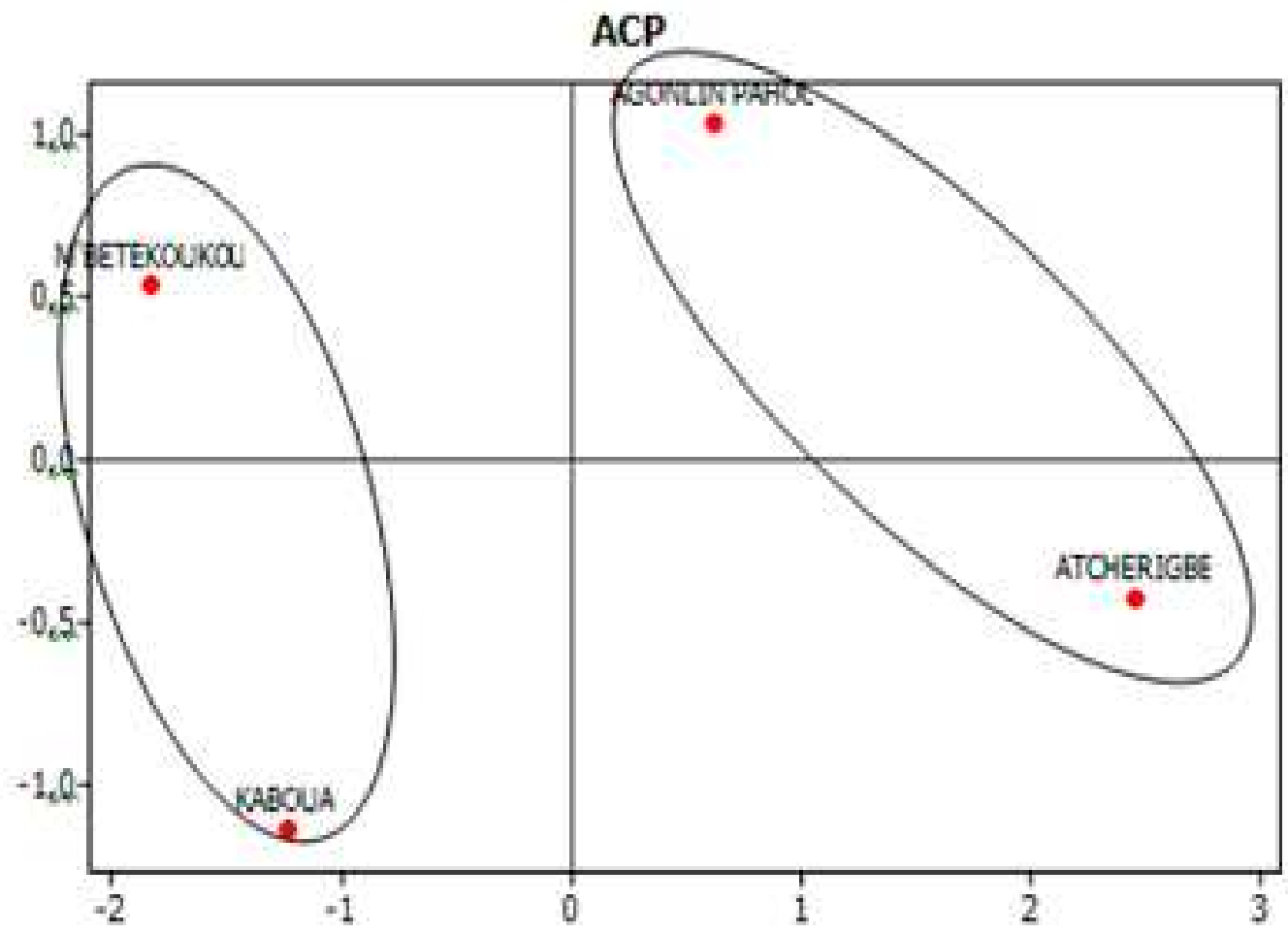

Figure 5 : Représentation des régions dans le système des deux premiers axes. ACP = Analyse en Composante principale.

Tableau 1: Formes d'occupation des sols dans la zone d'étude entre 1990 et 2008.

\begin{tabular}{lcccccc}
\hline & \multicolumn{2}{c}{ Année } & $\mathbf{1 9 9 0}$ & \multicolumn{2}{c}{ Année 2008 } & \multicolumn{2}{c}{ Variation } \\
\hline Forme d'occupation du sol & Ha & $\%$ & Ha & $\%$ & Ha & $\%$ \\
Galerie forestière & 39848 & 2.09 & 37048 & 1.94 & -2800 & -0.15 \\
Forêt dense & 17160 & 0.90 & 13430 & 0.70 & -63730 & -0.2 \\
Forêt claire et savane boisée & 263057 & 13.80 & 241357 & 12.66 & -21700 & -1.14 \\
Savane arborée et arbustive & 961344 & 50.43 & 590642 & 30.99 & -370702 & -19.44 \\
Savane saxicoles & 10866 & 0.57 & 10866 & 0.57 & 00 & 00 \\
Marécage & 24625 & 1.29 & 24625 & 1.29 & 00 & 00 \\
Cultures et de jachères & 446366 & 23.42 & 889260 & 46.65 & 442894 & 23.23 \\
Cultures et de jachères à palmiers & 105102 & 5.51 & 49878 & 2.62 & -55224 & -2.89 \\
Plantation & 9052 & 0,48 & 12502 & 0.66 & 3450 & 0.18 \\
Plan d'eau & 18906 & 0,99 & 18906 & 0.99 & 00 & 00 \\
Agglomération & 9834 & 0.52 & 17646 & 0.93 & 7812 & 0.41 \\
\hline
\end{tabular}


M.C. HOUEVOGANWA et al. / Int. J. Biol. Chem. Sci. 8(4): 1669-1683, 2014

Tableau 2 : Analyse de la variance de la prolifération du vecteur et des indices entomologiques et épidémiologiques par localité, dans le temps et par écosystème.

\begin{tabular}{|c|c|c|c|c|c|}
\hline Facteur & & $\mathbf{S}$ & TSI & MBR & PMT \\
\hline Source & Ddl & $\mathbf{F}$ & $\mathbf{F}$ & $\mathbf{F}$ & $\mathbf{F}$ \\
\hline Localité (L) & 3 & $4,86 * *$ & $0,43 \mathrm{~ns}$ & $13,71 * * *$ & $8,09 * * *$ \\
\hline Période(P) & 2 & $0,83 \mathrm{~ns}$ & $3,18 \mathrm{~ns}$ & $31,74 * * *$ & $1,35 \mathrm{~ns}$ \\
\hline Ecosystème(E) & 1 & $0,02 \mathrm{~ns}$ & - & - & - \\
\hline $\mathrm{R} \times \mathrm{P}$ & 6 & $0,55 \mathrm{~ns}$ & $0,19 \mathrm{~ns}$ & $1,52 \mathrm{~ns}$ & $2,52 *$ \\
\hline$P \times E$ & 2 & $1,15 \mathrm{~ns}$ & - & - & - \\
\hline $\mathrm{R} \times \mathrm{E}$ & 3 & $1,71 \mathrm{~ns}$ & - & - & - \\
\hline $\mathrm{R} \times \mathrm{P} \times \mathrm{E}$ & 6 & $1,86 \mathrm{~ns}$ & - & - & - \\
\hline
\end{tabular}

Tableau 3: Valeur moyenne des simulies et des indices parasitologiques par région. $(\mathrm{m}=$ moyenne ; se $=$ erreur standard $)$.

\begin{tabular}{|c|c|c|c|c|c|c|c|c|c|c|c|c|}
\hline \multirow{2}{*}{$\begin{array}{l}\text { Paramètre } \\
\text { Localités }\end{array}$} & \multicolumn{2}{|c|}{$\mathbf{S}$} & \multicolumn{2}{|c|}{ TSI } & \multicolumn{2}{|c|}{ MBR } & \multicolumn{2}{|c|}{ MTP } & \multicolumn{2}{|c|}{ SF } & \multicolumn{2}{|c|}{ SS } \\
\hline & $\mathbf{M}$ & Se & M & Se & M & Se & M & Se & $\mathbf{M}$ & Se & $\mathbf{M}$ & Se \\
\hline $\begin{array}{l}\text { Agonlin- } \\
\text { Pahou }\end{array}$ & $359,78 \mathrm{a}$ & 176,04 & $4,57 \mathrm{a}$ & 0,95 & $3256 a$ & 1549,33 & $146,42 \mathrm{ab}$ & 62,67 & $243,92 \mathrm{ab}$ & 94,80 & $115,85 a$ & 148,53 \\
\hline Kaboua & $1052,00 \mathrm{~b}$ & 176,04 & $5,23 \mathrm{a}$ & 0,95 & $9669 b$ & 1549,33 & $321,12 b c$ & 62,67 & $413,64 b c$ & 94,80 & $638,28 \mathrm{a}$ & 148,53 \\
\hline M'bétékoukou & $891,57 b$ & 176,04 & $4,02 \mathrm{a}$ & 0,95 & $8763 b$ & 1549,33 & $426,143 \mathrm{C}$ & 62,67 & 602,28 & 94,80 & $289,28 \mathrm{a}$ & 148,53 \\
\hline
\end{tabular}

$\mathrm{SF}=$ Simulies Forestière ; SS = Simulies Savanicoles. Sur la même colonne les chiffres de différentes lettres sont significativement différents (Test de Newman-Keuls). 


\section{DISCUSSION}

Ganglo et Henrix (1997) ont montré que les maigres ressources forestières disponibles en 1980 sont le résultat d'une dégradation des forêts par l'homme depuis plusieurs décennies et que de nos jours, les pressions humaines sur les forêts sont encore très fortes. On estime que chaque année au Bénin, environ 150000 ha de forêts disparaissent du fait de l'agriculture itinérante et des feux de brousse. Par ailleurs, Arouna et Sinsin (2002) ont prouvé que dans la forêt classée de l'Alibori Supérieur au Bénin, l'agriculture, l'élevage, le braconnage, l'exploitation forestière et l'usage des plantes médicinales ont eu pour effet, entre 1975 et 1998, une régression des galeries forestières, forêts claires, savanes boisées, savanes arborées et arbustives au profit des champs et jachères. Les travaux de ces auteurs confirment bien nos résultats. Cette dynamique spatio-temporelle de la végétation due aux activités humaines (abattage de la forêt et l'exploitation qui en découle, les feux de brousse) rend le milieu plus ouvert, fournit d'excellents gîtes de repos aux simulies dispersives et favorise considérablement leur dispersion à une saison où l'humidité relative est très élevée (Klein et Traore, 1984). Les simulies sont alors beaucoup plus attirées par les concentrations humaines, par suite des dégagements importants de gaz carbonique et de substances olfactives attractrices (Philippon, 1978). Par ailleurs, le recul progressif de la végétation supprime les massifs de forêts denses, qui constituaient des écrans à la dispersion des simulies sans toutefois réaliser une savanisation qui pourrait s'opposer efficacement aux déplacements simulidiens (Klein et Traore, 1984). D'autre part, le recul de la forêt est responsable de la réinvasion des zones par les simulies car facilite leur capacité de dispersion allant de $150 \mathrm{Km}$ jusqu'à $600 \mathrm{Km}$ de vol (Le Berre, 1966 ; Quillévéré, 1979). En effet, les simulies sont aidées par les vents de la mousson en occurrence le Front Intertropical (FIT) qui ne rencontre plus assez de difficultés au cours de son déplacement avec les simulies surtout dans les zones de savane. D'où la période de réinvasion d'avril/mai à juillet des zones forestières par les simulies savanicoles et vice-versa, facilitée par l'homogénéisation des caractéristiques physico-chimiques des eaux des gîtes larvaires (Quillévéré, 1979). Cette forte prolifération des simulies est responsable des taux mensuels de piqûres (TMP) élevés nettement supérieurs à la valeur maximale du taux annuel de piqûre (TAP) à partir de laquelle la nuisance simulidienne devient un problème de santé publique. Ces simulies provenant des zones périphériques sont en majorité des simulies pares (OMS, 1995), ce qui explique le PMT élevé surtout dans la zone Soudano-Guinéenne de Transition. Suite à cela, Prost et Rougemont (2009), ont démontré que des mesures environnementales et comportementales sont seules capables d'avoir un impact communautaire sur le niveau, la dispersion et la gravité de l'affection et que l'arrêt de la lutte anti-vectorielle en 2002 est synonyme d'une sous-estimation du potentiel de redispersion des simulies à partir des zones périphériques, et surtout l'impact de leur nuisance sur les populations rurales.

La disparition ou l'amputation de la forêt affecte la biologie et l'écologie des vecteurs donc crée de nouveaux écosystèmes favorisant surtout les vecteurs héliophiles (Andrew et al., 2001). A cet effet, certains de ces vecteurs sont en voie de disparition, d'autres s'adaptent, quelques uns prolifèrent. C'est le cas de A. gambiae S.S. qui prolifère en Afrique suite à la régression du couvert forestier. Par contre; la suppression des galeries forestières et la mise en culture des berges des cours d'eau (prophylaxie agronomique) ont été considérées, jusqu'en 1970, comme le moyen définitif pour éliminer 
les tsé-tsé et la maladie du sommeil (Mouchet et Carnevale, 1997). C'est ce phénomène qui explique certainement l'adaptation des simulies savanicoles et leur prolifération dans la zone d'étude au détriment des simulies forestières. Or Les migrations des simulies sont liées soit à la disponibilité de la nourriture des adultes, soit au préférendum physico-chimique des eaux des gîtes des stades préimaginaux. Les exigences des larves de simulies, en occurrence les Simulium damnosum s.s. constituent des facteurs limitants dans la répartition des différentes espèces (Quillévéré, 1979). Par conséquent, la régression de la forêt aurait donc eu des effets sur les caractéristiques physico-chimiques des eaux des gîtes larvaires favorisant l'installation des simulies savanicoles dans la zone d'étude.

\section{Conclusion}

L'étude de la dynamique spatiotemporelle des simulies, vecteurs de l'onchocercose cécitante en fonction de la dynamique de la végétation dans la zone d'étude révèle une forte régression du couvert forestier au profit des cultures et jachères, des plantations et des agglomérations; une forte prolifération des simulies par région dans le temps consécutif au recul temporel du couvert forestier avec un accent particulier dans le domaine Soudano-Guinéen de transition. Les simulies savanicoles (beaucoup plus porteuses des larves cécitantes) ont fortement accru ces dernières années dans chacune des régions, contrairement aux simulies forestières. Les régions de la zone Soudano-guinéenne sont beaucoup plus exposées à la résurgence de l'Onchocercose cécitante (PMT moyen élevé) et ses populations subissent plus de nuisance simulidienne par rapport à la zone Guinéenne.

Outre la distribution sous directive communautaire des comprimés d'ivermectine par le Programme National de lutte Contre les maladies Transmissibles (PNLMT), il urge que ce programme pense également à la lutte vectorielle contre la maladie sous toutes ses formes. Ceci dans l'intérêt surtout des riverains, compte tenu de la nuisance incompatible à toutes les activités agropastorales qu'ils subissent de la part des simulies et aussi du fait que les microfilaires d'Onchocerca volvulus sont en mesure de développer à tout moment une résistance à l'ivermectine; la dynamique de la population des simulies étant en faveur des simulies savanicoles. De plus, la caractérisation biologique des différentes souches de simulies et des microfilaires qu'elles hébergent, la promotion de nouvelles recherches scientifiques sur d'autres méthodes de lutte, moins coûteuses et moins toxiques pour l'environnement que les méthodes chimiques, s'avère nécessaire et pourrait participer à la lutte efficace contre cette maladie au Bénin.

\section{REFERENCES}

Agbahungba G, Sokpon N, Gaoué OG. 2001. Situation des ressources génétiques forestière du Bénin. Atelier sousrégional FAO/IPGRI/ICRAF sur la conservation, la gestion, l'utilisation durable et la mise en valeur des ressources génétiques forestières de la Zone Sahélienne (Ouagadougou 2224Sep. 1998). Note thématique sur les ressources génétiques forestières. Document FGR/12F. Département des forêts, FAO, Rome, Italie.

Anderson J, Fuglsang H, Hamilton PJS, Marshall TFC. 1974. Studies onchocerciasis in the United Cameroon Republic. II - Comparison of onchocerciasis in rain-forest and Sudansavanna. Trans. R. Soo. Trop. Med. Hyg., 68(3): 209-222.

Prost A, Rougemont A. 2009.Traiter les rivières pour vaincre une maladie sans intervention médicale: un défi relevé 
avec succès par l'OMS et les Universités. Rev Med Suisse, 5: 47-50.

Andrew KG, Steve WL, Ulisses EC, Jonathan AP. 2000. Changement climatique et maladies à transmission vectorielle : une analyse régionale. Bulletin of the World Health Organization., 78(9): 1136-1147.

Budden FH. 1963. Comparative study of ocular onchocerciasis in savanna and rainforest. Trans. Trop. Med., 57: 64-70.

Capo-chichi YJ. 2006. Monographie de la commune de Savè et de Dassa-Zounmè, Bénin.

Doukouré K, BissanY, Back C, Hougard JM, Agoua H, Guillet P, Konare M, Quilleveré D. 1994. Le programme de lutte contre l'onchocercose en Afrique de l'ouest : développement socioéconomique et risque de recrudescence de la transmission. Ann. Soc. Méd. Trop.,

74: $113-127$

FAO. 2009. Situation des Forêts du Monde. FAO: Rome, Italie ; 4.

FAO. 2010. Evaluation des ressources forestières mondiales 2010. Résultats principaux. FAO : Rome, Italie ; 3.

Ganglo JC, Henrix FM. 1997. Etat de la Recherche Forestière au Bénin - Bilan et Perspectives. XII World Forestly Congress : Québec city, Canada.

Harcel NT. 2008. La mouche noire et le comportement des populations, cas du bassin Nyong - Sanaga au Cameroun, mémoire de fin de formation. Mémoire rédigé en vue de l'obtention du diplôme d'Ingénieur d'Application de la Statistique, Institut Sous-régional de Statistique et d'Économie Appliquée, Yaoundé, p. 69.

Klein JM, Traoré LM. 1984. Mission entomologique sur la nuisance des simulies dans la région de Makondo (sanaga maritime). Rapport, Institut Français de recherche scientifique pour le développement en coopération, Yaoundé, p. 17.

Le Berre R. 1966. Contribution à 1'étude biologique et écologique de Simulium damnosum Theobald, 1903, (Diptera: Simuliidae). Mém. ORSTOM, $\mathrm{n}^{\circ}$ 17, p. 204.

Leonard O. 1996. Contribution à l'élaboration d'un modèle de Transmission de l'Onchocercose dans le foyer de M'BAM (Cameroun). D.U. de $3^{\text {ème }}$ cycle de Cartographie, Université de Montpellier III, Monpellier, p. 75.

Monjusiau AGM, Lagraulet J, d'Haussy J, Cöckel CW. 1965. Aspect ophtalmologique de l'onchocercose au Guatemala et en Afrique Occidentale. Bull.Org. Mond. Santé., 32: 339-355.

Mouchet J, Carnevale P. 1997.Impact des transformations de l'environnement sur les maladies à transmission vectorielle. Cahiers Santé., 7: 263-269.

OMS. 1995. Succès en Afrique, le programme de lutte contre l'onchocercose en Afrique de l'Ouest. OCP/GVA.

OMS. 1998. Vingt années de lutte contre l'onchocercose. OCP/GVA.

Ousséni A, Sinsin B, Houndagba CJ. 2002. Exploitation des ressources biologiques et la dynamique de la forêt classée de l'Alibori supérieur au Bénin (secteur de l'arrondissement de Bagou). Mémoire de maîtrise soutenu au Département de Géographie et Aménagement du Territoire, FLASH/ UAC, Bénin, Abomey-Calavi, p. 117.

Philippon B. 1977. Etude de la transmission d'onchocerca volvulus Leuckart 1903 (Diptera: Simulidae) en Afrique tropicale. Trav. et Doc. De l'ORSTOM $\mathrm{N}^{\circ} 63$, p. 308.

Philippon B. 1978. L'Onchocercose Humaine en Afrique de l'Ouest: Vecteur, Agent Pathogène, Epidémiologie et Lutte. ORSTOM : Paris ; 198. 
Poinsignon A. 2008. Etude de la relation homme-vecteur. De l'identification à la validation de protéines salivaires comme marqueur immunologique d'exposition aux piqûres d'Anopheles spp et de Glossina spp. Thèse de doctorat, Université Montpellier I, Montpellier, p. 228.

Quillévéré D. 1979, Contribution à l'étude des caractéristiques taxonomiques, bioécologiques et vectrices des membres $\mathrm{du}$ complexe Simulium damnosum présents en Côte d'Ivoire. Trav. et Doc. ORSTOM, Paris, p. 304.

Traoré S, Enyong P, Mandiangu ML, Kayembé D, Zouré H, Noma M, Sékétéli A. 2006.Transmission d'Onchocerca volvulus par Simulium damnosum à Inga (République démocratique du Congo). Cahier d'Etudes et de Recherche Francaphones/Santé, 16(2) : 77-82. 